\title{
CIENCIAS SOCIALES

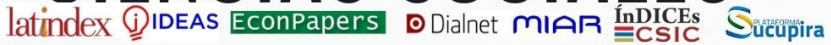

\section{LOS MODELOS DE CALIDAD COMO CRITERIO DE EXCELENCIA, EL MODELO EFQM APLICADO A LA EDUCACIÓN}

\author{
Elena González-Fenoll \\ Docente en ISEN Centro Universitario. Universidad de Murcia. \\ https://orcid.org/0000-0002-4014-6878 \\ e.gonzalezfenoll@um.es \\ Abraham Bernárdez-Gómez \\ Contratado Predoctoral (BES-2017-081040). Universidad de Murcia. \\ https://orcid.org/0000-0003-1862-5554 \\ abraham.bernardez@um.es
}

Para citar este artículo puede utilizar el siguiente formato:

Correspondencia: abraham.bernardez@um.es

Elena González-Fenoll y Abraham Bernárdez-Gómez: "Los modelos de calidad como criterio de excelencia, el modelo EFQM aplicado a la educación", Revista Caribeña de Ciencias Sociales (Especial noviembre 2021, pp. 49-61) En línea:

https://doi.org/10.51896/caribe/PTYD3669

\section{RESUMEN}

La búsqueda de la calidad y mejora dentro de las organizaciones, ha llevado a trasladar desde el mundo de la empresa diversas herramientas para que ello se produzca, también, en el ámbito educativo. En el presente texto se ofrece un recorrido por el conocimiento y la investigación actual sobre el modelo de calidad EFQM y cómo se desarrolla su dinámica de funcionamiento y mejora en los centros. Se ha elaborado a partir de una investigación bibliográfica, de corte cualitativo $y$, mediante un análisis de contenido se ha tratado de dar por desarrollado el objetivo establecido para este trabajo: analizar y establecer una visión global sobre el modelo de calidad EFQM. Podemos establecer como principales conclusiones la, aún presente, reticencia de los centros o modificar las dinámicas que en ellos se desarrollan puesto que exige un esfuerzo y una mirada hacia lo propio muchas veces incómoda. Sin embargo, queda patente cómo la aplicación de modelos de este tipo redunda de forma sustancial en la mejora de dichos procesos.

Palabras clave: EFQM, calidad, mejora, centros educativos.

\section{QUALITY MODELS AS A CRITERION OF EXCELLENCE, THE EFQM MODEL APPLIED TO EDUCATION.}

\section{ABSTRACT}

The search for quality and improvement within organizations has led to transfer from the world of the company various tools for them to occur, also, in the educational field. This text offers a tour of 
current knowledge and research on the EFQM quality model and how its dynamics of operation and improvement in the centers are developed. It has been prepared based on a qualitative literature research and, through a content analysis, an attempt has been made to develop the objective established for this work: analyze and establish a global vision on the EFQM quality model. We can establish as main conclusions the, still present, reluctance of the centers or modify the dynamics that develop in them since it demands an effort and a look towards the own often uncomfortable. However, it is clear how the application of models of this type substantially results in the improvement of these processes.

Keywords: EFQM, quality, improvement, educational centers.

\section{INTRODUCCIÓN}

El siguiente artículo tiene como objetivo analizar y establecer una visión global sobre el modelo de calidad EFQM. Los modelos de calidad surgieron en 1991 como fruto de los exitosos resultados de diversas organizaciones $y$, aunque su fin original era estar destinados a empresas, organizaciones de elevada índole como la Comisión Europea, la Organización Europea para la Calidad y la Asociación Europea para la Gestión de la Calidad, han procurado que este factor se extienda a los organismos educativos (Martínez y Riopérez, 2005; Machorro et al. 2016).

En la actualidad, son cada vez más las instituciones que utilizan los modelos de excelencia, en particular, el Modelo de Excelencia de la Asociación Europea para la Gestión de la Calidad, a partir de ahora EFQM, debido a que es una herramienta de gestión directa y completa que permite llevar a cabo el proceso de autoevaluación de las organizaciones de manera eficiente, para alcanzar la calidad total de las mismas (Gómez et al., 2014).

Las aportaciones que derivan de estos modelos son beneficiosas para las estructuras de los centros educativos ya que potencian el análisis de sus fortalezas y debilidades (Belmonte y Bernárdez-Gómez, 2021; Bernárdez-Gómez et al., 2021), la autoevaluación frente a modelos más genéricos, sustentan que se produzca una mejora continua y permiten mostrarse ante otras organizaciones externas (Machorro, 2012; Tamayo et al., 2011).

Uno de los principales objetivos de las instituciones educativas es la calidad en la educación que imparten, entendida ésta como un constructo funcional, eficaz y eficiente que permite cumplir las metas inicialmente propuestas a través del uso competente de sus recursos (Martínez y Riopérez, 2005). Si trasladamos esta idea al sujeto que recibe dicha educación, el alumno, la actual ley orgánica de educación en España 3/2020, de 29 de diciembre, por la que se modifica la Ley Orgánica 2/2006, de 3 de mayo, de educación (LOMLOE) afirma que para que la educación de un país sea de calidad debe asegurar y transmitir, entre otras cuestiones, los conocimientos, habilidades, capacidades y disposiciones necesarias para que el alumnado alcance la vida adulta de la manera más saludable posible y formando así, individuos comprometidos con la sociedad. 
Algunos de los factores fundamentales del EFQM que atañen a los centros fomentando que éstos obtengan resultados positivos son: la orientación al cliente, en este caso, el alumnado, su familia y por consiguiente la sociedad; liderazgo y constancia para la consecución de los objetivos propuestos; el desarrollo y participación de todos los miembros; gestionar los procesos y los resultados alcanzados; fomentar la ampliación de las alianzas de cara a obtener nuevos y más actualizados proveedores así como establecer, dentro de la organización, un proceso continuo de aprendizaje y formación permanente posibilitando la innovación y la mejora (Adalid, 2019).

Consciente de ello, son cada vez más los centros educativos que utilizan el EFQM para evaluarse y por tanto, conocer su situación actual a fin de elaborar planes de mejora que les permitan alcanzar sus metas (Tirado y Conde, 2015; Espiñeira-Bellón et al., 2016).

Todo ello, unido a los Objetivos de Desarrollo Sostenible propuestos dentro de la Agenda 2030 de Desarrollo Sostenible, reflejan una mirada al pasado para construir un nuevo futuro más consciente y preocupado con el planeta (Fábregas, 2019; Romero, 2019).

Por tanto, a la vista de los acontecimientos, son muchas las investigaciones que muestran el concepto del EFQM y las implicaciones que presenta hacia la consecución de la calidad total dentro de los centros escolares (Cantón y Barrios, 2015; Conde et al., 2015; Sánchez et al., 2015; Grau y Trilla, 2017).

En las siguientes partes del presente estudio, se encuentra desarrollada la parte metodológica referente a las investigaciones de este tipo, un apartado de análisis de resultados y discusión donde se exponen los principales productos de la tarea realizada y, un último espacio donde se expresan las principales conclusiones obtenidas.

\section{METODOLOGÍA}

Como se había advertido, en el presente trabajo se realizará una revisión bibliográfica para dar cumplimento al objetivo establecido en la introducción: Analizar y establecer una visión global sobre el modelo de calidad EFQM.

Una vez recogidos los documentos, se ha procedido a una profundización que, en palabras de Bisquerra (2016), debe ser sistemática con la finalidad de que sirva a generar conocimiento. Así, para realizar dicho análisis, nos hemos servido del programa de análisis cualitativo ATLAS.ti en el cual se han generado un total de 6 categorías de análisis que servirán para establecer una visión holística de lo que es el modelo EFQM. Las mencionadas categorías atienden a la estructura formal establecida en este tipo de documentos, siendo éstas: conceptualización, objetivos del modelo, aplicación y composición, criterios de excelencia, fundamentos y cambios que origina.

Para llegar al mencionado objetivo se ha realizado una revisión sistemática que, según Orteso y Caballero (2017), es un proceso a realizar en cuatro etapas. Establecer los criterios para la búsqueda, descargar los documentos y un primer análisis de los documentos descargados para 
emplear los que cumplan los criterios de selección y posteriormente, una categorización final de los textos.

Revisiones como las que se desarrollan en el presente documento hacen referencia a investigaciones de tipo secundario (Cea D’Ancona, 1996; Pacios, 2013) dónde se lleva a cabo un análisis de contenido (Miles et al., 2014) como un proceso en el que se deben tener en cuenta elementos de la teoría fundamentada para su codificación (Alarcón et al., 2017). Realizando una primera codificación abierta, con el fin de identificar los distintos conceptos subyacentes; una segunda axial, determinando las relaciones que nos podemos encontrar; y una última codificación selectiva, con el fin de analizar los datos de forma conjunta.

\section{RESULTADOS Y DISCUSIÓN}

El modelo de Excelencia EFQM tiene la misión de lograr la excelencia en la gestión de las organizaciones para que sirvan de modelo a seguir por otras instituciones (Pastor et al., 2014; Rivera, 2018). Es un modelo no normativo de autoevaluación, y se basa en criterios preestablecidos -por tanto, es un modelo criterial- que se explica en apartados siguientes.

EI EFQM dota a las organizaciones de un sistema de autogestión siempre orientado a la mejora de la organización (Zárraga-Rodríguez et al., 2014; Martínez et al., 2018).

Este Modelo de Excelencia fue introducido en 1991 en España como base de los premios de calidad, entre ellos el Premio Europeo de Calidad; que trata de estimular y asistir a las organizaciones para participar en actividades de mejora enfocadas a lograr la excelencia en la satisfacción del cliente, la satisfacción de los empleados, el impacto en la sociedad y en los resultados de negocio, fortaleciendo de este modo la posición competitiva de las empresas europeas en los mercados mundiales (Ortíz y Rúa, 2017). A nivel educativo, el Ministerio de Educación y Ciencia lo adoptó en 1997 como proceso para la mejora, el diagnóstico y la autoevaluación del sistema educativo. Así, concretamente el Modelo EFQM Sector Público es un medio ordenado y sistemático de autoevaluación, que posibilita la reflexión sobre ella y la elaboración de planes estratégicos de mejora, y evaluación interna para la mejora de la calidad de los servicios (Martínez-Moreno y Díaz, 2017).

Para ello, se establecen una serie de objetivos que se deben cumplir para seguir este modelo que, como se puede observar, están centrados en las dinámicas de centro y a su vez, establece cómo éstas se pueden mejorar y desarrollar para alcanzar mayores niveles de calidad dentro de las organizaciones. Dentro de los objetivos que encontramos en este modelo cabría destacar:

- Desarrollar metas y visión para el futuro de las organizaciones.

- Identificar y entender la naturaleza de la misma, las relaciones de sus actores y las relaciones causa-efecto. 
- Establecer un mismo lenguaje y modo de pensar en toda la organización.

- Diagnosticar los puntos de mejora e implementar acciones que ayuden a dicha mejora.

- Reconocer a las organizaciones mejor gestionadas y promoverlas como modelos de excelencia.

\section{Aplicación y Composición del Modelo de Excelencia EFQM}

Para alcanzar dichos objetivos, se debe llevar a cabo un proceso de aplicación sistemática y periódica del Modelo de Excelencia EFQM por parte del Equipo directivo en un Centro Educativo que facilita tres cuestiones fundamentales dentro del desarrollo del modelo. El establecimiento de planes de mejora continuos que sirven como misión y visión conjunta para todos los profesionales del centro y que marquen un camino de acción hacia el que se tiene que dirigir la comunidad educativa (Juaneda et al., 2013). La fundamentación de esos planes de mejora debe estar traducida en hechos completamente objetivos, establecidos en base a unas necesidades palpables y, así estar basados en la realidad en la que está inmersa el centro acrecentando, por tanto, la visión común sobre las metas a alcanzar ya mencionadas y las herramientas a utilizar (Carrillo et al., 2018).

Así, la aplicación del Modelo de Excelencia EFQM está basado en la Comprensión profunda del modelo por parte de todos los niveles de dirección del Centro Educativo y la Evaluación de la situación del mismo en cada una de las áreas.

A su vez, el Modelo de Excelencia EFQM consta de dos partes:

1. Conjunto de criterio de Excelencia, las cuales abarcan todas las áreas de funcionamiento del Centro Educativo. Son en total un conjunto de 9 criterios divididos en dos grupos: Agentes y Resultados.

1.1. Agentes (1-5): pretenden evaluar aspectos del sistema de gestión del Centro Educativo. Son la causa de los resultados. Se denominan: liderazgo, política y estrategia, personas, alianzas y recursos, procesos.

1.2. Resultados (6-9): se refiere a lo que el Centro Educativo consigue para cada uno de sus actores (alumnado, familias, comunidad). Se denominan: resultados en los clientes, resultado en las personas, resultados en la sociedad y resultado clave.

Para cada grupo de criterio hay un conjunto de reglas de evaluación basadas en la llamada "lógica REDER" que explicaremos más adelante.

2. Conjunto de reglas para evaluar el comportamiento del Centro Educativo en cada uno de los criterios. 


\section{Criterios de Excelencia Educativa}

El Modelo EFQM Educación está compuesto por 9 criterios, cinco de los cuales son facilitadores y los restantes cuatro de resultados. En relación con estos criterios, se evalúa el progreso del establecimiento educacional en su camino hacia la excelencia (Villa et al., 2015; Moreno, 2016; Suárez et al., 2017).

1. Liderazgo. Los coordinadores tienen la capacidad para dirigir y motivar, de manera eficiente, las acciones de la organización hacia el logro de las metas comunes propuestas. El equipo directivo del colegio debe ser accesible a todo el personal y se hace necesario una normativa para la toma de responsabilidades, la valoración y la incentivación de la participación

2. Política y estrategia. Son los medios de los que se vale la organización para lograr los objetivos propuestos. Definir la política y la estrategia para todos los procesos del centro educativo es fundamental y, concretarlos con objetivos claros, así como, determinar procedimientos para el seguimiento de logros e indicadores para su medición y valoración. La responsabilidad y la coordinación es entre todos.

3. Personas. Se refiere a la participación del personal en todos los niveles. Aprovechando la experiencia, promoviendo la innovación y facilitando la expresión de la creatividad en todas sus formas. Se elaboran planes de formación para los profesores en tecnologías y enfoques didácticos. Diseñar y aprobar un plan de evaluación interna de los profesores. Incentivación del personal para conseguir proyectos beneficiosos para el centro.

4. Alianza y recursos. Enfatiza cómo planificar y gestionar la organización las alianzas externas y sus recursos internos en apoyo de su política y estrategia. Obteniendo recursos mediante concursos a proyectos, premios, colaboraciones con administraciones locales y colaborar con los centros educativos del entorno.

5. Procesos. Trata de cómo diseñar, gestionar y mejorar los procesos de la organización para satisfacer plenamente a los usuarios. Designando responsables de procesos y estableciendo procedimientos para su gestión, definiendo sus objetivos y concretando las estrategias.

6. Resultados en los usuarios. Qué logros está alcanzando la organización en relación a sus usuarios. Destacando positivamente la disponibilidad de todo el personal para atender a padres y alumnos.

7. Resultados en las personas. Las organizaciones de excelencia miden de manera exhaustiva los resultados que esperan que se alcancen en las personas que la integren. Existiendo una buena relación del personal con los alumnos y los padres, realizando convenios para participar en la formación y ampliar las relaciones con otras instituciones.

8. Resultados en la sociedad. Las organizaciones de excelencia miden de manera exhaustiva 
los resultados que esperan que se alcancen en la sociedad. Explorando las necesidades y expectativas de la sociedad en relación con el centro, mejorando las instalaciones del centro y ofrecerlas al entorno próximo.

9. Resultados Clave. Las organizaciones de excelencia miden de manera exhaustiva los resultados que esperan que se alcancen con respecto a los elementos clave de su política y estrategia. Se valora de modo positivo el cumplimiento de los horarios, de las programaciones didácticas y de la gestión del material y equipamientos.

Los agentes han de tener un enfoque bien fundamentado e integrado con otros aspectos del sistema de gestión, su efectividad ha de revisarse periódicamente con objeto de reflexionar, aprender y mejorar, y han de estar sistemáticamente desplegados e implantados en las operaciones de la organización afín de enaltecer la imagen y calidad del centro educativo (Montiel, Méndez y López, 2011).

La excelencia se logra cuando los resultados satisfacen a todos los grupos implicados en la organización (Martínez-Moreno y Díaz, 2017). Las mejoras propuestas deben estar fundamentadas en información fiable, que incluya los hechos y las percepciones de los miembros del establecimiento educacional y de sus clientes. Los resultados han de mostrar tendencias positivas, compararse favorablemente con los objetivos propios y con los resultados de otras organizaciones, estar causados por los enfoques de los agentes y abarcar todas las áreas relevantes (Ortíz y Rúa, 2017).

\section{Fundamentos del Modelo EFQM}

En los fundamentos del modelo se encuentra un esquema lógico que se denomina REDER (En inglés RADAR) que es una herramienta que permite evaluar el rendimiento de la institución y que está formada por cuatro elementos (Ruiz et al., 2016). Este esquema lógico establece lo que un centro necesita realizar como, por ejemplo, determinar los Resultados que quiere lograr formando parte del proceso de elaboración de su política y estrategia. Estos resultados cubren el rendimiento de la organización, tanto en términos económicos y financieros como operativos, así como las percepciones de todos los grupos de interés de la organización. También se debe planificar y desarrollar una serie de Enfoques sólidamente fundamentados e integrados que la lleven a obtener los resultados requeridos ahora y en el futuro. Desplegar los enfoques de manera sistemática para asegurar una implantación completa. Finalmente, es necesario Evaluar y Revisar los enfoques utilizados basándose en el seguimiento y análisis de los resultados alcanzados y en las actividades continuas de aprendizaje. En función de todo ello, identificar, establecer prioridades, planificar e implantar las mejoras que sean necesarias.

Al utilizar el Modelo en un centro, por ejemplo, para realizar una autoevaluación, los elementos Enfoque, Despliegue, Evaluación y Revisión del esquema lógico REDER deben abordarse en cada subcriterio de grupo "Agentes Facilitadores", y el elemento "resultados" debe abordarse en cada subcriterio del grupo "Resultados". 
La redacción de la memoria debe incluir en todo momento la lógica REDER dónde el centro debe evaluar cada uno de los cinco criterios del grupo de agentes facilitadores, de acuerdo con los cuatro elementos (Acuña, 2012):

1. Enfoque

2. Despliegue

3. Evaluación

4. Revisión

Cada uno de ellos, incorpora a la evaluación diferentes atributos. El enfoque contiene los métodos que el centro emplea para implantar el criterio. El centro deberá tener en cuenta durante la realización de la autoevaluación y la elaboración de la memoria atributos como la solidez de enfoque y la integración.

En un centro con una organización excelente, el enfoque será:

1. Con fundamento claro: es decir, con una lógica clara centrada en las necesidades del centro (actuales y futuras).

2. Con procesos bien definidos y desarrollados, enfocado claramente a los actores.

3. Integrado, con apoyo en la política y la estrategia y adecuadamente enlazado con otros enfoques.

El despliegue constituye la traslación a la práctica de lo especificado en el enfoque. Si la implantación está alineada y ha sido sistemática, nos da a entender que la política y la estrategia del centro están presentes en el despliegue habitual de los diferentes enfoques y en todos los niveles de la organización del centro.

Para la evaluación del despliegue, el centro deberá tener en cuenta durante la realización de la autoevaluación y la elaboración de la memoria los siguientes atributos:

- Sistemático: alcanza hasta qué punto se gestiona el despliegue de forma estructurada.

- Implantado: incluye hasta qué punto se ha implantado el enfoque en las áreas más importantes del centro y en todos los procesos del centro.

Evaluación y revisión. En este elemento se aborda lo que hace el centro para revisar el enfoque, mejorarlo y desplegarlo en todas sus áreas y niveles. Periódicamente y de forma sistemática, deberán revisarse el enfoque, el despliegue del enfoque y los resultados obtenidos.

El centro deberá tener en cuenta durante la realización de la autoevaluación y la elaboración 
de la memoria los siguientes atributos:

- Medición: evaluar el grado de consecución de los resultados deseados.

- Aprendizaje: identificar las mejores prácticas y las áreas de mejora (benchmarking, evaluaciones de rendimiento etc.).

- Mejora: cómo el centro utiliza los resultados de la medición y la mejora para implantar y priorizar áreas de mejora.

Los resultados en el Modelo EFQM se refieren a los logros que el centro está alcanzado. En un centro con una organización excelente según Espiñeira-Bellón, Mato y Mariño (2016) se tendrán en cuenta factores como las tendencias positivas y los enfoques adoptados por el centro o un buen nivel sostenido de acuerdo con los resultados obtenidos; si los objetivos son adecuados y se alcanzan; la comparativa de los resultados favorablemente con los de otros, en especial con los mejores y si su alcance cubre todas las áreas relevantes para los actores (grupos de interés) así como la segmentación de éstos para que se identifiquen y entiendan mejor las oportunidades de mejora.

El centro deberá tener en cuenta durante la realización de la autoevaluación y la elaboración de la memoria las siguientes cualidades: Los resultados presentan tendencias positivas y si el rendimiento alcanzado es bueno y sostenido; el grado de consecución de los objetivos planificados y si éstos son buenos para el centro; las comparaciones con otros centros u organizaciones excelentes en su gestión; el grado en que los resultados son consecuencia del enfoque; el porcentaje de áreas de la organización que obtienen buenos resultados.

\section{Cambios que origina la Excelencia en la gestión}

El establecimiento de este modelo de gestión supone una diferencia importante con respecto a la idea tradicional. Se encamina a alcanzar la excelencia del centro educativo que los adopte, enfocada en evolucionar desde la antigua conceptualización hasta otra donde prima la calidad (Piqueras, 2017).

- La dirección del centro mantendrá una visión de desarrollo y de éxito para lograr la excelencia.

- La política del centro se plantea hacia el grupo de interés, los alumnos.

- Anteriormente la calidad era asunto de la dirección, con este modelo la calidad concierne y se garantiza por todos los empleados del centro, para los que se busca extraer todo su potencial.

- De lo anterior se desprende que la organización va a actuar de forma cohesionada y no desfragmentada como hasta ahora. Tratando de que la participación sea unos de los ejes principales de actuación. 
- El cambio en el entorno se pasa a entender como una necesidad que ha de cubrir el centro.

- El tipo de gestión se transforma gracias a los indicadores, los cuales señalan las oportunidades de mejora.

\section{CONCLUSIONES}

Como conclusiones principales acerca de los beneficios que supone utilizar el modelo EFQM en los centros educativos para realizar el proceso de autoevaluación y por tanto, conocer las posibles carencias y buscar en la medida de lo posible, la mejora como fin para alcanzar el éxito educativo, podemos resaltar de acuerdo al análisis DAFO y en base a sus debilidades, que en dichos centros existe cierta reticencia al cambio de los agentes implicados así como falta de experiencia, escasa disponibilidad de tiempo por parte de los equipos directivos y docentes para su implantación y por consiguiente, resistencia a la evaluación tanto de los órganos docentes como de los propios centros en general. En la literatura citada (Cantón y Barrios, 2015; Conde et al., 2015; Sánchez et al., 2015; Espiñeira-Bellón et al., 2016; Grau y Trilla, 2017), es frecuente comprobar la existencia real de que, en la mayoría de los centros educativos, se sigue estableciendo un sistema tradicional en el que el cuerpo docente, opta por no enfrentarse a las nuevas demandas que genera el alumnado, fruto de la limitada actualización y formación de este. Como amenazas posibles se puede comprobar que, este hecho, provoca que las organizaciones educativas, no innoven en su práctica docente y por tanto, no se produzcan mejoras que fomenten entre otras cuestiones, una educación inclusiva que llegue a todo el alumnado. No obstante, y resaltando lo anteriormente expuesto, en muchas ocasiones la falta de conocimientos acerca del modelo o la gran demanda de tiempo que genera, así como determinados conceptos abstractos que complican su implementación, se constituyen como factores fundamentales a la hora de accionar el modelo EFQM como mecanismo interno de evaluación de las instituciones educativas.

Sin embargo, las principales fortalezas que este modelo ofrece están relacionadas con la sencillez de uso, la consciencia real acerca de la propia Comunidad Educativa favoreciendo su reflexión sobre su práctica diaria, la combinación entre evaluación externa para conocer qué están haciendo las demás instituciones y el autoconocimiento acorde a la consecución de los objetivos propuestos, la posibilidad de coexistir con otros modelos de evaluación, el protagonismo global de todos los recursos tanto humanos, como espaciales y materiales del centro, la obtención de resultados que posibilitan la mejora y el éxito mediante la implantación de un plan de Calidad, el cambio de un sistema tradicionalista a un sistema más actual e inclusivo en el que se transformen las actitudes y prácticas produciéndose un modelo más positivista e innovador.

Para finalizar, este modelo genera una serie de oportunidades como la investigación-acción ya que permite que lo expuesto mediante la autoevaluación, no se quede en un mero trámite teórico sino que favorece que los resultados se lleven a la práctica; la mejora del clima y la Cultura Escolar, una mayor estructuración de los procesos que se producen en la enseñanza y el aprendizaje; la innovación en la Comunidad Educativa así como la mejora de todos los agentes de la misma; la 
posibilidad de descubrir las fortalezas y debilidades de la organización fundamentadas en hechos y datos como base para los planes de mejora así como una mayor cohesión de todo el personal en torno al proyecto educativo del centro.

\section{REFERENCIAS}

Acuña, J. E. (2012). Modelo de gestión de calidad académica para la educación superior. Calidad en la educación superior, 3(1), 157-184.

Adalid, P. (2019). La gestión por procesos en la mejora continua de centros educativos. Quaderns digitals: Revista de Nuevas Tecnologías y Sociedad, 88, 142-162.

Alarcon, A., Munera, L. y Montes, A. (2017). La teoría fundamentada en el marco de la investigación educativa. Saber, ciencia y libertad, 12(1), 236-245.

Belmonte Almagro, M. L., \& Bernárdez-Gómez, A. (2021). Evaluation of Self-Concept in the Project for People with Intellectual Disabilities: "We Are All Campus". Journal of Intelligence, 9(4), 50. https://doi.org/10.3390//intelligence9040050

Bernárdez-Gómez, A., González Barea, E.M. \& Rodríguez Entrena, M.J. (2021). Educational Reengagement Programs and Measures in the Region of Murcia. An Approach to their Understanding. International Journal of Sociology of Education, 10(3), 246-270. http://doi.org/10.17583/rise.7516

Bisquerra, R. (2016). Metodología de la investigación educativa. La Muralla.

Cantón, I. y Barrios, N. (2015). Calidad de las escuelas bolivarianas de Venezuela. Fuentes: Revista de la Facultad de Ciencias de la Educación, 16, 155-172.

Carrillo, F. X., Carrillo, V. H. y Moreno, C. J. (2018). Calidad total. Un enfoque de la administración del siglo XXI. RECIMUNDO, Revista Científica de la Investigación y el Conocimiento, 2(3), 634647.

Cea D’Ancona, M. A. (1996). Metodología Cuantitativa. Estrategias y técnicas de investigación social. Síntesis.

Conde, S., Azaustre, M. C. y Delgado, M. (2015). Análisis integral de la gestión de la convivencia escolar. Una propuesta de evaluación. International Journal for 21st Century Education (IJ21CE), 2(1), 39-59.

Espiñeira-Bellón, E. M., Mato, D. y Mariño, M. C. (2016). Análisis descriptivo del impacto de Sistemas de Gestión de Calidad (EFQM e ISO) en centros de Educación Primaria de la Comunidad Autónoma Gallega. Revista electrónica interuniversitaria de formación del profesorado, (19)3, 103-113.

Fábregas, S. (2019). Las características del nuevo modelo EFQM 2020. Forum Calidad, 306, 40-44.

Gómez, J., Martínez-Costa, M. y Martínez-Lozano, A. R. (2014). Los modelos de excelencia como herramientas de gestión. Revista de contabilidad y dirección, 19, 29-49.

Grau, A. y Trilla, J. (2017). El sistema de evaluación del desempeño en la Diputación de Tarragona: Un instrumento de desarrollo profesional. Pertsonak eta Antolakunde Publikoak Kudeatzeko Euskal Aldizkaria = Revista Vasca de Gestión de Personas y Organizaciones Públicas, 12, 42-51. 
Jiménez, J. A., Martínez, M. y Jiménez, D. (2019). Proposal of an empirical model that integrates relationships between organizational culture, learning and quality management. [Jornadas Doctorales]. Universidad de Murcia.

Juaneda, E., González, L. y Marcuello, C. (2013). El reto de la calidad para el Tercer Sector Social. Análisis de casos de implantación del modelo EFQM. Cuadernos de gestión, 13(2), 111-126.

Ley orgánica 3/2020, de 29 de diciembre, por la que se modifica la Ley Orgánica 2/2006, de 3 de mayo, de educación. (2020). Boletín Oficial del Estado, 340, de 30 de Diciembre de 2020, 122868-122953. https://www.boe.es/boe/dias/2020/12/30/pdfs/BOE-A-2020-17264.pdf

Machorro, F. (2012). Autoevaluación de la Excelencia Administrativa en una Institución de Educación Superior Tecnológica del Estado de Veracruz. ConCiencia Tecnológica, 44, 10-14.

Machorro, F., Mercado, P., Cernas, D. A. y Romero, M. V. (2016). Influencia del capital relacional en el desempeño organizacional de las instituciones de educación superior tecnológica. Innovar, revista de ciencias administrativas y sociales, 26(60), 35-50.

Martínez, A. y Díaz, A. (2017). Evaluar la gestión de la calidad en los servicios deportivos municipales de la región de Murcia. Modelo EFQM. Journal of sport and health research, 9(3), 301-310.

Martínez-Moreno, A. y Díaz, A. (2017). Uso del modelo EFQM como contraste del nivel de gestión en la calidad de los servicios deportivos municipales. Revista Euroamericana de Ciencias del Deporte, 6(1), 101-106.

Martínez, P, Pérez, F. J. y Martínez, M. (2018). Aplicación de los modelos de gestión de calidad a la tutoría universitaria. Revista complutense de educación, 29(3), 633-649.

Martínez, C. y Riopérez, N. (2005). El modelo de excelencia de la EFQM y su aplicación para la mejora de los centros educativos. Educación XXI, 8, 35-66.

Miles, M. B., Huberman, A.M. y Saldaña, J. (2014). Qualitative Data Analysis. A Methods Sourcebook. Sage.

Montiel, E., Méndez, D. y López, A. (2011). Propuesta de autoevaluación de las organizaciones educativas. Gestión de la educación, 1(1), 1-27.

Moreno, L. (2016). El proceso de evaluación según el modelo EFQM: una experiencia en la Biblioteca Universitaria de Granada. Boletín de la asociación andaluza de bibliotecarios, 31(112), 84100.

Orteso, P. y Caballero, C. M. (2017). Educación intercultural, experiencias inclusivas: un recorrido por el siglo XXI. Interacções, 43, 254-276.

Ortíz, J. M. y Rúa, A. (2017). Gestión de la calidad y diseño específico de los procesos de admisión en el sistema universitario español: Estudio de caso en una universidad privada. REICE: Revista Iberoamericana sobre Calidad, Eficacia y Cambio en Educación, 15(1), 87-106.

Pacios, A. (2013). Técnicas de búsqueda y uso de la información. Editorial Universitaria Ramón Areces.

Pastor, A. C., Pastor, J., Navarro, L., Sodhi, M. y Pérez, G. Creating a holistic excellence model adapted for technology-based companies. Tourism and Management Studies, 10, extra 1, 718. 
Piqueras, M. (2017). La EFQM como modelo integral. Consultor de los ayuntamientos y de los juzgados, revista técnica especializada en administración local y justicia municipal, 5, 585595.

Rivera, F. (2018). Influencia del Modelo Europeo de Excelencia en la cultura de la organización. Journal of Healthcare Quality Research, 33(4), 193-198.

Romero, M. (2019). Modelo EFQM: la gestión excelente, innovadora y sostenible en el ámbito sanitario. Forum Calidad, 307, 17-19.

Ruiz, A., Cuerva, V., Cabeza, D. y Roldán, M. I. (2016). Modelo EFQM y organismo autónomo de entidad local. ¿Compatibilidad?. Cuadernos de estudios empresariales, 26, 33-60.

Sánchez, A. V., Ruiz, P. E. T., y Ruiz, F. D. (2015). Estructura latente y fiabilidad de las dimensiones que explican el impacto de los sistemas de gestión de calidad en los centros educativos. Revista de investigación educativa, RIE, 33(1), 65-82.

Suárez, E., Calvo-Mora, A., Roldán, J. L. y Periáñez-Cristóbal, R. (2017). Quantitative research on the EFQM excellence model. A systematic literature review (1991-2015). European Research on Management and Business Economics, 23(3), 147-156.

Tamayo, P. F., Moreno, M. R., Ochoa, M. C., De León, I. S. y Arteta, Y. (2011). Los enfoques mundiales de la gestión de la calidad. Una mirada desde los modelos y premios de excelencia. Ingeniare, 11, 99-113.

Tirado, R. y Conde, S. (2015). Análisis estructural de la convivencia escolar desde el modelo de la European Foundation Quality Management (EFQM). Educatio siglo XXI, (33)1, 195-214.

Villa, A., Troncoso, P. E. y Díez, F. (2015). Estructura latente y fiabilidad de las dimensiones que explican el impacto de los sistemas de gestión de calidad en los centros educativos. Revista de investigación educativa, 33(1), 65-82.

Zárraga-Rodríguez, M., Suárez-Barraza, M. F., Jaca, C., Álvarez, M. J. y Viles, E. (2014). Information capability under different quality management approaches. Revista de globalización, competitividad y gobernabilidad, 8(3), 33-44. 